\title{
LINEAR METRIC SPACES AND ANALYTIC SETS
}

\author{
by ROBERT KAUFMAN*
}

(Received 19th January 1993)

\begin{abstract}
A problem in descriptive set theory, in which the objects of interest are compact convex sets in linear metric spaces, primarily those having extreme points.
\end{abstract}

1991 Mathematics subject classification: 54H05, 28A05.

\section{Introduction}

Let $E$ be a linear metric space with norm $\|x\|$ and distance $d(x, y)=\|x-y\|$, and suppose that $E$ is separable and complete. The space $c(E)$ of all non-void compact subsets of $E$ is then a Polish space in the Hausdorff metric, and its topology depends only on that of $E$. The space $\mathscr{K}(E)$ of all compact, convex (non-void) subsets of $E$ is closed in $c(E)$; we are interested primarily in the subset $\mathscr{E} \mathscr{K}(E) \subseteq \mathscr{K}(E)$, consisting of those sets in $\mathscr{K}(E)$ having at least one extreme point. A remarkable example by Roberts $[4,5,9]$ shows that $\mathscr{E} \mathscr{K}(E) \neq \mathscr{K}(E)$ when $E=L^{1 / 2}(0,1)$ for example.

We push this a step further:

Theorem. $\mathscr{E} \mathscr{K}(E)$ is always an analytic subset of $\mathscr{K}(E)$, and is a complete analytic subset when $E=l^{2} \oplus L^{1 / 2}$.

The second part of the theorem means this: for each analytic set $A$ in a compact metric space $M$, there is a continuous mapping $\phi$ of $M$ into $\mathscr{K}(E)$, such that $A=\phi^{-1}(\mathscr{E} \mathscr{K}(E))$. Previous work on extreme boundaries of convex sets in Banach spaces, and on the class of (non-compact) convex sets admitting extreme points, is presented in $[2,3,6,7]$; applications of Roberts' technique appear in $[1,10]$.

\section{The basic construction}

Let $K_{0}$ be a compact, convex set in $L^{1 / 2}$ containing 0 but no extreme point, and $K_{1}$ a compact, convex set in a Banach space, say $l^{2}$. (In the space $l^{2} \oplus L^{1 / 2}$, the subspace $l^{2} \oplus(0)$ is identified with $l^{2}$, and likewise for $\left.(0) \oplus L^{1 / 2}\right)$. We define a sort of extension of $K_{1}$ by $K_{0}$, parametrized by a function $f \geqq 0$ in $C\left(K_{1}\right)$. Let $L(f)$ be the compact subset of $K_{1}+K_{0}$ containing all $(x, y)$ such that

* Research supported in part by the National Science Foundation. 


$$
x \in K_{1}, y \in f(x) \cdot K_{0}
$$

and

$$
K(f)=\overline{c o}(L(f)) \subseteq K_{1}+\|f\|_{\infty} \cdot K_{0}
$$

Lemma 1. $K(f)$ has an extreme point if and only if $f$ has a zero in ex $K_{1}$.

This depends on an elementary result, whose proof is omitted.

Lemma 0. Let $C$ be a compact convex set in $E, F$ a closed subset of $C$, and $z$ and extreme point of $\overline{c o}(F)$. Then there is an element $w$ of $F$, such that $z \in \overline{c o}(F \cap V)$ for every neighbourhood $V$ of $w$.

We apply this with $C=K_{1}+\|f\|_{\infty} \cdot K_{0}$, and $F=L(f)$. Let $z=(x, y)$ be an extreme point of $K(f)$. The element $w$ of $L(f)$ must have the same first co-ordinate as $z$ (since $l^{2}$ is locally convex), so we write $w=\left(x, y_{1}\right), y_{1} \in f(x) \cdot K_{0}$. To see that $y \in f(x) \cdot K_{0}$, let $\delta>0$ and let $V$ be a neighbourhood of $\left(x, y_{1}\right)$ such that $f(\xi)<f(x)+\delta$ whenever $(\xi, \eta) \in V$. Then $\overline{c o}(V \cap L(f)) \subseteq K_{1}+(f(x)+\delta) \cdot K_{0}$; since $K_{0}$ is compact, and $\delta>0$ was arbitrary, we conclude that $y \in f(x) \cdot K_{0}$.

Continuing with the consideration of $z \in e x K(f)$, we see that it is necessary that $f(x)=0$, whence $y=0$. But then it is necessary as well as that $x \in e x K_{1}$, i.e. $f^{-1}(0)$ meet ex $K_{1}$.

Conversely, suppose that $x \in e x K_{1}$ and $f(x)=0$, and $2(x, 0)=\left(x_{1}, y_{1}\right)+\left(x_{2}, y_{2}\right)$, with $\left(x_{i}, y_{i}\right) \in K(f)$. Clearly $x_{1}=x_{2}=x$, and we proceed to prove that $y_{1}=y_{2}=0$. There is a formula $\left(x, y_{1}\right)=\lim \int(x, y) d \mu_{n}(x, y)$, where each $\mu_{n}$ is an atomic probability measure in $L(f)$, with finite support. Writing $c_{n}=\int f(x) d \mu_{n}$, and $c=\liminf c_{n}$, we see that $y_{1} \in c \cdot K_{0}$. Let $\mu$ be any $w^{*}$-limit of the sequence $\left(\mu_{n}\right)$, so that $x=\int x d \mu(x, y)$-since $l^{2}$ is a Banach space. This is possible only if $\mu$ is concentrated on $(x) \oplus L^{1 / 2}$, and thus $\lim c_{n}=0$. We have proved that $y_{1}=0$, i.e. $(x, 0)$ is an extreme point, thereby proving Lemma 1 .

A small observation is necessary: $K(f)$ depends continuously on the parameter $f \in C^{+}\left(K_{1}\right)$. Indeed, when $\|f-g\|_{\infty}<\delta$, then $f \leqq g+\delta$, so $K(f) \subseteq K(g+\delta) \subseteq K(g)+\delta \cdot K_{0}$. This implies continuous dependence of $K(f)$ on $f$.

\section{An elementary step}

Let $F_{m}$ be the subset of $E \times \mathscr{K}(E)$ defined as follows:

$$
(x, K) \in F_{m} \quad \text { if } \quad x+y, x-y \in K
$$

for some $y$, such that $\|y\| \geqq m^{-1}$. (Here $m=1,2,3, \ldots$ ). To see that $F_{m}$ is closed in $E \times \mathscr{K}(E)$, suppose $\left(x_{n}, K_{n}\right) \in F_{m}$, and $\lim x_{n}=x, \lim K_{n}=K$. Then $x_{n} \pm y_{n} \in K_{n}$, for some $y_{n}$ with $\left\|y_{n}\right\| \geqq m^{-1}$. Now $\bigcup_{1}^{\infty} K_{n}$ has compact closure in $E$, whence $\left(x_{n}+y_{n}\right)$ has a 
limit-point, and so $\left(y_{n}\right)$ has a limit-point $y$. Thus $\|y\| \geqq m^{-1}$ and $x+y, x-y$ belong to $K:(x, K) \in F_{m}$.

The union $\bigcup_{1}^{\infty} F_{m} \equiv F$ is exactly the set of pairs $(x, K)$ such that $x \in K \backslash$ ex $K$. Projecting the $G_{\delta}$-set $E \times \mathscr{K}(E) \backslash F$ on the second factor $\mathscr{K}(E)$, we obtain an analytic set, namely $\mathscr{E} \mathscr{K}(E)$.

\section{A special set in $l^{2}[8]$}

Let $C$ and $C_{0}$ be the subsets of $l^{2}$ containing sequences $\left(a_{k}\right)_{1}^{\infty}$ such that $\sum k a_{k}^{2} \leqq 1$ (respectively, $\sum k a_{k}^{2}=1$ ). Then $C$ is compact and convex, and $C_{0}=e x C$. The remaining part of the proof is based on the next observation (zero-set representation):

(ZSR). Let $A$ be an analytic set in a compact metric space $M$. There is a continuous function $f(t, x) \geqq 0$ on $M \times C$ such that $t \in A \Leftrightarrow f(t, x)=0$ for some $x \in C_{0}$.

The conclusion of the main argument is presented next, and then finally the proof of $Z S R$. We use $C$ in place of $K_{1}$. The partial function $x \mapsto f(t, x)$ is denoted $f_{t}$, and then $M$ is mapped into $\mathscr{K}\left(l^{2} \oplus L^{1 / 2}\right)$ by the formula $K[t]=K\left(f_{t}\right)$. Then $K[t]$ has an extreme point $\Leftrightarrow f_{t}$ has a zero in $C_{0} \Leftrightarrow t \in A$. We have seen that $K\left(f_{t}\right)$ depends continuously on $f_{t}$, i.e. $K[t]$ varies continuously with $t$. This proves the main result.

Proof of ZSR. A certain detail makes this appear complicated. The mapping of a function $g \in C([0,1])$ to its zero set $g^{-1}(0)$ is merely upper semicontinuous; but this can be detoured by enlarging the domain $[0,1]$. The set $A$ is the image $\psi(N)$ of the set $J$ of irrationals in $I=[0,1]$ by a continuous function $\psi$ defined over $J$. Let $\Gamma$ be the graph of $\psi$-thus $\Gamma \subseteq J \times A$-and let $d$ be a metric for $I \times M, 0 \leqq d \leqq 1$. Then each $t$ in $M$ is mapped to a closed set $F(t)$ in $I \times M \times I$ : the set of all 3-tuples $(s, t, d((s, t), \Gamma))$. This is continuous and $t \in A \Leftrightarrow F(t)$ meets $J \times M \times(0)$, a $G_{\delta}$-set in $I \times M \times I$. (The device just introduced is the detour mentioned above.)

Next we define a map $h$ from the cube $Q=[0,1]^{N}$ to $C$ such that $h^{-1}\left(C_{0}\right)=(0,1]^{N}$. The triangular function $\delta(u)=\max (1-|u|, 0)$; for each $\lambda \geqq 1$ there is a scalar $c(\lambda)>0$ such that $c(\lambda) \delta(n-\lambda)$ belongs to $C_{0}$ (as a function of $n$ ), and we call this $g(\lambda)$, defining $g(+\infty)=0$. Finally $h\left(s_{1}, s_{2}, s_{3}, \ldots\right)=\sum_{1}^{\infty} 2^{-k / 2} g\left(s_{1}^{-1} \cdots s_{k}^{-1}+3 k\right)$. It is a simple matter to adapt this to an arbitrary metric space $Q$ and any $G_{\delta}$ subset $V$, in particular $Q=I \times M \times I$ and $V=J \times M \times(0)$. Hence $H(t) \equiv h(F(t))$ is a closed subset of $C$, $t \in A \Leftrightarrow H(t)$ meets $C_{0}$; now we conclude by defining $f(t, x)=d(x, H(t))$.

\section{REFERENCES}

1. J. Bourgain and H. P. Rosenthal, Martingales valued in subspaces of $L^{1}$, Israel J. Math. 37 (1980), 54-75.

2. R. HAYDON, A new proof that every Polish space is the extreme boundary of a simplex, Bull. London Math. Soc. 7 (1975), 97-100.

3. J. E. J Jyne and C. A. Rogers, The extremal structure of convex sets, J. Funct. Anal. 26 (1977), 251-288. 
4. N. J. Kalton and N. T. Peck, A re-examination of the Roberts example of a compact convex set without extreme points, Math. Ann. 253 (1980), 89-101.

5. N. J. Kalton, N. T. Peck and J. W. Roberts, An F-space Sampler (London Math. Soc., Lecture Notes 89, 1984).

6. R. Kaufman, Co-analytic sets and extreme points, Bull. London Math. Soc. 19 (1987), 72-74.

7. R. Kaufman, Extreme points and descriptive sets (manuscript).

8. E. T. Poulsen, A simplex with dense extreme points, Ann. Inst. Fourier (Grenoble) 11 (1961), 83-87.

9. J. W. Roberts, A compact convex set with no extreme points, Studia Math. 60 (1971), 255-266.

10. M. Talagrand, Un espace de Banach réticulé qui a presque la proprieté de RadonNikodým, Studia Math. 76 (1983), 255-266.

Department of Mathematics

University of Illinois at Urbana-Champaign

1409 West Green Street

URBANA

ILLINOIS 61801

USA 